Research Article

\title{
Wireless Resource Management and Resilience Optimization of the M2M-Oriented Mobile Communication System
}

\author{
Li Liao $\mathbb{B D}^{1,2}$ and Chengjun Ji ${ }^{1}$ \\ ${ }^{1}$ School of Business Administration, Liaoning Technical University, Huludao, Liaoning 125105, China \\ ${ }^{2}$ School of Economics and Management, Zhejiang University of Science and Technology, Hangzhou 310013, China \\ Correspondence should be addressed to Li Liao; liaoli@zust.edu.cn
}

Received 1 November 2021; Revised 24 November 2021; Accepted 29 November 2021; Published 17 December 2021

Academic Editor: Gengxin Sun

Copyright (C) $2021 \mathrm{Li}$ Liao and Chengjun Ji. This is an open access article distributed under the Creative Commons Attribution License, which permits unrestricted use, distribution, and reproduction in any medium, provided the original work is properly cited.

\begin{abstract}
Machine-to-machine (M2M) communication technology is an emerging technology that can connect smart wireless devices. The most obvious feature of $\mathrm{M} 2 \mathrm{M}$ is that the communication between devices does not require human intervention. Therefore, ensuring the low-energy consumption of $\mathrm{M} 2 \mathrm{M}$ devices is a necessary condition for prolonging the survival time of their devices. This paper first considers the coexistence of $\mathrm{M} 2 \mathrm{M}$ and $\mathrm{H} 2 \mathrm{H}$ scenarios. Aimed at the energy efficiency of $\mathrm{M} 2 \mathrm{M}$ equipment and the channel capacity of $\mathrm{H} 2 \mathrm{H}$ equipment, a multiobjective problem is constructed for joint spectrum and power resource management, and a weighted Chebyshev algorithm is proposed to solve this problem. Secondly, in view of the additional interference problems caused by the introduction of $\mathrm{M} 2 \mathrm{M}$ communication, the intercell cooperative link selection algorithm is used to optimize its resilience. The effectiveness of the algorithm is proven by simulation results.
\end{abstract}

\section{Introduction}

The development of wireless communication technology and people's needs complement each other. Compared with the existing 4G (4th generation) technology, the upcoming 5G (5th generation) technology will provide higher transmission rate, lower transmission delay, and higher user satisfaction [1, 2]. Machine-to-machine communication (M2M) technology is not only the key component of Internet of things technology but also the basis of realizing information society. Therefore, this paper focuses on M2M technology $[3,4]$.

M2M uses sensor and other technologies to enable wireless communication equipment to have the ability of autonomous communication [5]. The biggest feature of this technology is that it does not need human intervention. Therefore, it can be applied to a variety of scenarios, such as intelligent transportation system, intelligent monitoring system, medical system, public security monitoring, and electronic meter reading [6]. These application scenarios are composed of a large number of mobile devices, and different devices have different quality of service, which will cause huge traffic consumption. According to literature [7], it is predicted that the total number of connections of M2M devices in 2022 will increase to 18 billion compared with the current total number of connections of M2M devices, accounting for $61 \%$ of the total accessible communication devices. Therefore, in the face of such a large number of M2M devices, people have paid more and more attention to their size, power, and cost. M2M technology and human-to-human communication (HTC) technology are different. The research focus of HTC technology is mainly to improve the user's transmission rate and reduce the delay, and the number of devices is far less than the number of M2M devices. When MTC devices and HTC devices coexist in the same cellular network and compete for spectrum resources together, the traditional communication quality between people will be affected by MTC. Therefore, in the future $5 \mathrm{G}$ ultradense M2M scenario and the network where MTC and HTC coexist, how to ensure the performance of MTC equipment without affecting the performance of HTC equipment is an important problem to be solved at this stage [8]. 
At the same time, in order to meet the QoS requirements of such large-scale M2M devices, it is necessary to effectively allocate the current wireless resources. Therefore, this paper proposes a new wireless resource management scheme. Secondly, for the additional interference caused by the introduction of $\mathrm{M} 2 \mathrm{M}$ communication, it is necessary to provide corresponding wireless resource management and interference coordination strategies to optimize the system performance.

\section{Related Knowledge}

2.1. M2M. The network architecture of M2M specified by the technical committee of the European Telecommunications Standards Association is shown in Figure 1. M2M transfers data from one terminal to another, that is, the dialogue between machines. For example, access control cards for work, bar code scanning in supermarkets, and NFC mobile payment are more popular recently. Key M2M elements in the figure include the following: M2M device, $\mathrm{M} 2 \mathrm{M}$ area network (device domain) providing connection between the M2M device and M2M gateway, personal local area network, M2M gateway using the M2M function to ensure interworking and interconnection between the $\mathrm{M} 2 \mathrm{M}$ device and communication network, M2M communication network providing communication between the M2M gateway and M2M application, and M2M application program included in the application layer [9]. For M2M applications, their data passes through various applications and is processed and used by a specific business processing engine. M2M equipment forms an M2M regional network. Its applicable scenarios include not only small home networks but also larger scenarios, such as factory environment.

For the network architecture in Figure 1, the M2M device can be connected to the M2M server through WAN connection or M2M gateway. A gateway is an intelligent $\mathrm{M} 2 \mathrm{M}$ device, which can easily collect and process data from $\mathrm{M} 2 \mathrm{M}$ devices and manage their operations.

\subsection{Research on M2M Wireless Resource Management. Dif-} ferent from the traditional communication system, in $\mathrm{M} 2 \mathrm{M}$ communication, a large number of devices with multiple quality of service requirements access the network, which brings great challenges to resource allocation. At present, a large number of literatures have studied the radio resource management of $\mathrm{M} 2 \mathrm{M}$ communication which is different from $\mathrm{H} 2 \mathrm{H}$ communication, mainly including access control, spectrum allocation, and power control. This paper mainly focuses on spectrum allocation and power control [10].

At present, there are many possible architectures for M2M communication. Document [11] proposes three architectures, including direct communication between M2M devices and ENB, multihop transmission by gateway, and end-to-end transmission between M2M devices.

Because the uplink communication adopts single carrier frequency division multiple access (FDMA) multiple access mode, when M2M equipment communicates directly with $\mathrm{ENB}$, the allocated frequency domain resource blocks are

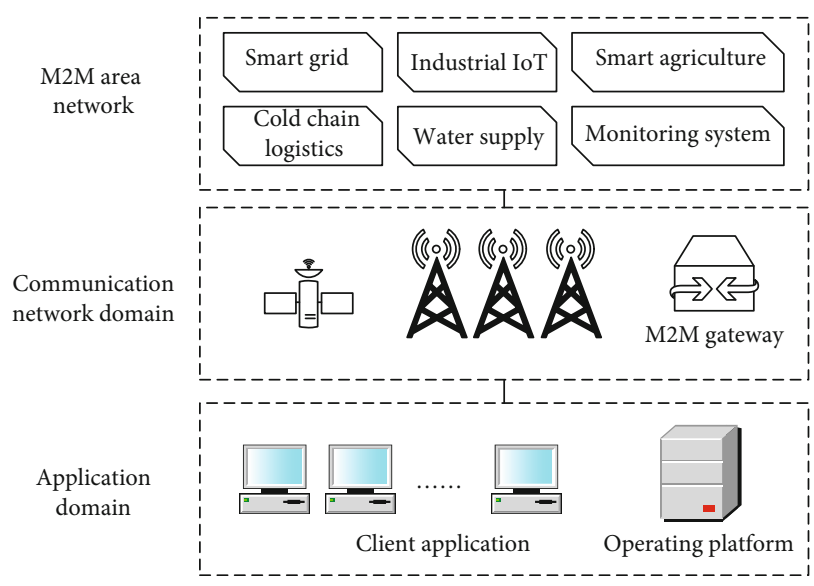

Figure 1: Network architecture of M2M.

required to be continuous, which increases the difficulty of resource allocation. C-FDMA single-carrier frequency division multiple access (single-carrier frequency division multiplexing) is the mainstream multiple access in the uplink of LTE. SC-FDMA is a single-carrier modulation mode. The basic processing methods can be divided into $\mathrm{dft}$-s-gmc and dft-s-ofdm. In Reference [12], resource block allocation is transformed into resource block mode allocation, and the optimization problem is solved by combining the Lagrange dual method and ellipsoid method. According to the multiservice characteristics of M2M equipment, document [13] divides resource allocation into two steps. Firstly, the equipment is graded according to the equipment type and the allowable length of residual delay, and the equipment that can be scheduled is selected. Then, the appropriate resource block is selected according to the channel conditions of the equipment in different frequency bands. In Reference [14], user terminal equipment (UE) and M2M equipment are put into two queues, respectively, and the calculation formula of proportional fair scheduling algorithm is improved. This method not only considers the current channel conditions but also takes into account QoS and waiting delay. Based on this, they proposed a new scheduling algorithm. Both algorithms give M2M devices lower priority and ensure the performance of $\mathrm{H} 2 \mathrm{H}$ communication when a large number of M2M devices join. However, in the case of heavy $\mathrm{H} 2 \mathrm{H}$ load, these two methods can easily make it difficult for M2M equipment to communicate for a long time.

According to the mass characteristics of M2M devices, packet transmission of M2M devices is a good idea. Each section of information, together with the call control signal and verification information required for exchange, is arranged into a message packet according to the specified format. A packet is transmitted as a whole in the network, with high utilization of communication resources, which greatly improves the capacity and throughput of the channel. Document [15] proposes to group M2M devices and uses the traditional uplink spectrum resource allocation method for spectrum selection between groups and ordinary $\mathrm{H} 2 \mathrm{H}$ devices. Device-to-device (D2D) communication is adopted in the group, and nonorthogonal spectrum is used with $\mathrm{H} 2 \mathrm{H}$ equipment. In order to reduce the interference 
of M2M equipment to $\mathrm{H} 2 \mathrm{H}$ equipment, the whole problem is modeled as a bipartite graph, and the $\mathrm{KM}$ algorithm is used to allocate resources. Reference [16] uses a similar architecture, uses the $\mathrm{KM}$ algorithm to realize resource allocation in the case of $\mathrm{M} 2 \mathrm{M} / \mathrm{H} 2 \mathrm{H}$ shared spectrum, and performs power control based on the interference level reflected by the proportional integral derivative (PID) controller. However, at present, most M2M devices do not support D2D communication, resulting in limited application scenarios. Document [17] proposes a nonorthogonal resource allocation method. M2M devices conduct random access in groups. Devices belonging to different groups can use the same group of resource blocks to improve spectral efficiency and adopt a continuous interference cancellation method to reduce interference. Literature [18] combines access control and resource allocation to reduce energy consumption and completes the determination of grouping, team leader selection, resource allocation, and packet size.

In addition, M2M equipment can also communicate with UE. Reference [19] proposed a distributed resource allocation method. Using the matching principle, the M2M device can negotiate with the UE device and give the UE certain compensation in exchange for the access of the subchannel. Document [20] proposes to use UE as the gateway of M2M equipment. In the first stage, the M2M device transmits the information to the corresponding UE. In the second stage, the UE transmits the information of the M2M device and its own information to the base station. By solving the optimization problem and setting the appropriate transmission power, the power consumption of M2M equipment is minimized on the premise of meeting the delay constraint, so as to improve the network lifetime of M2M equipment.

2.3. Optimize Interference and Signal Fading. Signal fading [21] and interference [22-24] are two important problems encountered in the design of the mobile communication system. Fading results in the reduction of coverage and reliability of point-to-point communication links. The existence of interference limits the reusability of wireless resources, thus limiting the improvement of spectral efficiency (BPS/Hz/area) per unit area [25]. In view of these two important problems, combined with the development process and trend of the mobile communication network, this section summarizes the research status of the M2M mobile communication scenario and the development trend of related mobile communication technologies.

As shown in Figure 2, in the mobile communication network based on intercell cooperation, it is assumed that the base stations are connected to each other through a backhaul network without delay and capacity limitation. Under this assumption, the base stations can share the global channel state information and all data information of their users. In this scenario, multibase station cooperation actually regards the whole multicell network as a large multiuser multiple input multiple output (MIMO) system [26, 27]. Therefore, the multibase station joint signal processing algorithm can eliminate interference, so as to greatly improve the system capacity [28-30].

\section{Method}

3.1. M2M Resource Allocation Algorithm. In the scenario of direct connection between MTC equipment and base station, MTC equipment and HTC equipment share the same frequency band resources, resulting in resource competition between the two system networks. At the same time, this paper maximizes the energy efficiency of MTC equipment and the rate of HTC equipment, so as to complete the reasonable allocation of bandwidth and power resources.

The system model diagram of this paper is shown in Figure 3. Suppose that there is only one base station (ENB) in this cell, and there are $n$ MTC devices and M HTC devices in its coverage. These devices are randomly distributed in the cell, where the set of MTC devices is $\Omega=\{1,2, \cdots, n\}$ and the set of HTC devices is $\Xi=\{1,2, \cdots, m\}$. In this paper, it is assumed that all devices are directly connected to the base station for data transmission.

In this cell model, since the communication behavior of MTC equipment transmitting data mainly occurs in the uplink, the uplink is considered the main link. It is assumed that all access devices work in orthogonal frequency bands, so the interference between devices is ignored. The total shared bandwidth of all devices is set to $B_{\max }$, and this paper assumes that the base station can obtain the global channel side information (CSI) of all devices. In this paper, the gain between the MTC device $n$ and the base station can be expressed as

$$
d_{n}^{\alpha} g_{n}=h_{n} .
$$

Similarly, the gain between the HTC device $m$ and the base station is expressed as

$$
d_{m}^{\alpha} g_{m}=h_{m} .
$$

The signal propagating at multipath can reach the simultaneous interpretation of the field strength at the receiving point from different paths, and the delay time of each path is different. The superposition of the component waves in each direction produces a standing wave field strength, thus forming a fast fading signal, called Rayleigh fading, where $\mathrm{HN}$ represents that the variable has experienced slow fading and Rayleigh fading on the transmission link, DN represents the distance between MTC user $n$ and the base station, and $\alpha$ is the path loss index.

In order to balance the performance of MTC devices and HTC devices, we will construct a multiobjective optimization problem. Compared with the single-objective optimization problem which can only obtain the unique solution, it is more suitable to solve the Pareto optimal solution in the coexistence scenario of MTC and HTC. Therefore, the weighted Chebyshev method is used in this paper.

The signal-to-noise ratio (SNR) of MTC equipment can be written as

$$
p_{n}^{-1} \gamma_{n}=N_{0}^{-1} b_{n}^{-1} g_{n}
$$

Here, $\quad p_{n} \geq 0$ and $b_{n} \geq 0$ represent the allocated 


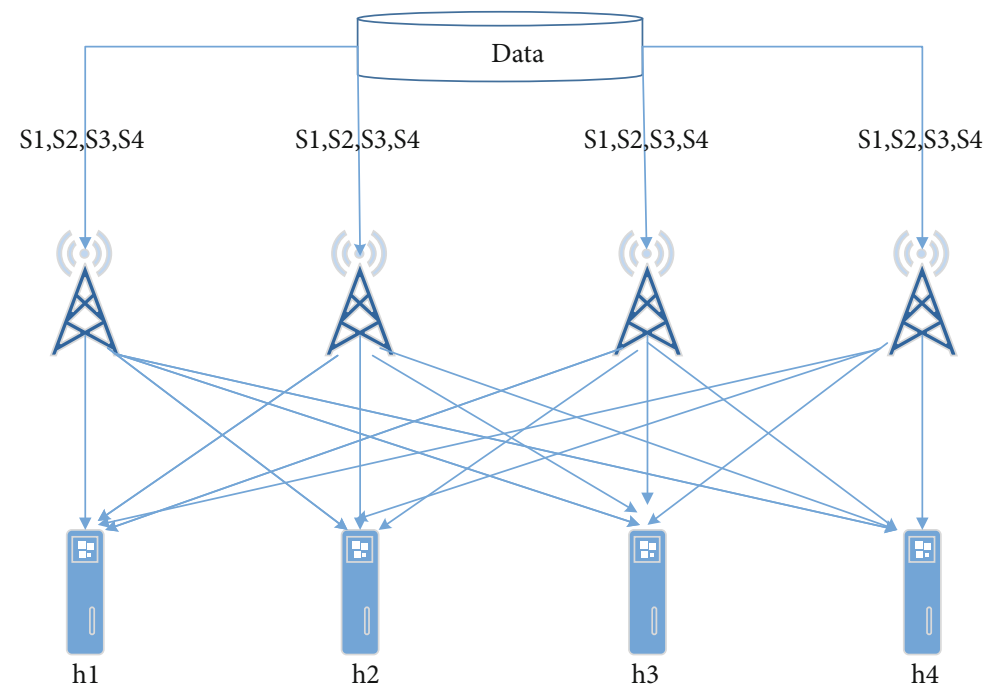

Figure 2: Cooperative multicell system model.

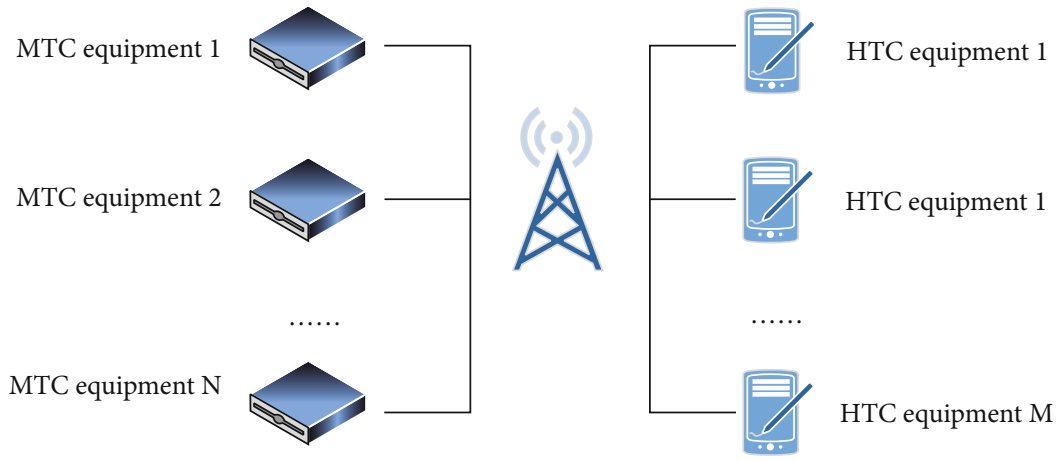

FIGURE 3: System model.

transmission power and bandwidth of MTC device $n$ in the uplink, respectively, and $n$ represents the power spectral density of additive white Gaussian noise (AWGN).

Therefore, the energy efficiency (EE) of a single MTC device can be expressed as

$$
e_{n}=p_{n}^{0} \log _{2}\left(\gamma_{n}+1\right) b_{n} .
$$

Here, $r_{n}$ represents the transmission bit rate from MTC device $n$ to the base station. In addition, $p_{n}^{0}=p_{n}+p_{e}$, where $p_{e}$ represents the fixed power consumption.

For HTC equipment, the uplink rate is also an important indicator in the future 5G communication. Therefore, the channel capacity requirements of HTC equipment are the focus of consideration. According to Shannon's theorem, the bit rate of HTC equipment $m$ in the uplink can be written as follows:

$$
r_{m}=b_{m} \log _{2}\left(N_{0}^{-1} b_{n}^{-1} g_{n}+1\right) .
$$

$p_{m}=\left\{p_{1}, p_{2}, \cdots p\right\}$ and $b_{m}=\left\{b_{1}, b_{2}, \cdots, b\right\}$ are the optimized power and bandwidth variables of HTC devices, respectively. $r_{m}$ represents the uplink bit rate of each HTC device, and $b_{m}$ and $p_{m}$ represent the bandwidth and power allocated to each HTC device, respectively. $g_{m}$ represents the gain between HTC device $m$ and base station.

Before introducing the weighted Chebyshev algorithm, this section first needs to deal with the weight factor accordingly. When a large number of MTC devices are connected to the cell network, more subcarriers are allocated to MTC devices, which will seriously affect the QoS of HTC devices. To solve this situation, this paper defines the ratio of the total number of subcarriers required by MTC devices to the bandwidth of the whole network The value is its demand factor $(\mathrm{DF}) p$. Then, the weight function of energy efficiency of MTC equipment $\Phi_{1}(\rho)$ can be defined as follows:

$$
\phi_{1}(\rho)=(1-\exp s p)(1-\exp s)^{-1} .
$$

Here, $s$ is a parameter greater than 1 , which affects the curve slope of the weight function, and is set here as $\Phi_{1}(\rho) \in[0,1]$, and the objective function increases with the increase in $p$. The gradient is a decreasing function, which shows that the sensitivity of the energy efficiency objective 


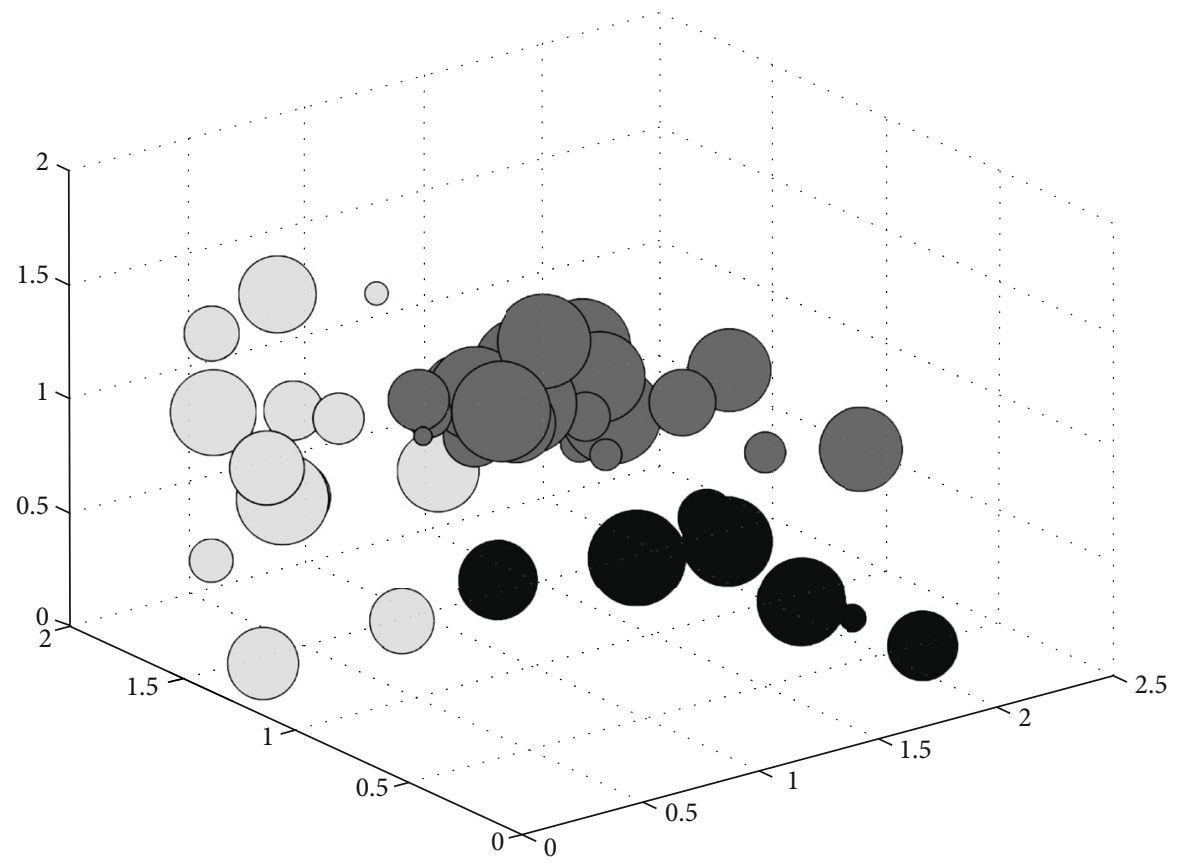

Figure 4: Cooperative multicell network layout and user distribution.

function of MTC equipment to a high DF factor is reduced. The energy consumption problem of MTC terminal can be solved by the communication unit deciding whether to enter the transmission state according to the relationship between the current channel signal-to-noise ratio and the preset threshold, which is obtained based on the maximum value of the energy efficiency function of MTC terminal. In addition, the weight function expression of HTC equipment can be calculated by $\Phi_{2}(\rho)=1-\Phi_{1}(\rho)$.

For the two optimization objectives, finding the minimum value is the core idea of the weighted Chebyshev algorithm.

$$
\min _{\{p, b\}} \max _{n \in \Omega}\left\{\phi_{1}-f_{1}^{u} \phi_{1} \sum r_{n} p_{n}^{-1}, \phi_{1}-f_{2}^{-u} \phi_{2} \sum r_{m}\right\}
$$

Here, $\Phi_{1}$ and $\Phi_{2}$ represent the positive weight factors of energy efficiency and system capacity functions, respectively. For the sake of fairness, the value of each single objective function is divided by its ideal point for normalization.

3.2. Optimization of Interference and Fading Signals. OFDMA combines OFDM and FDMA technology. After subcarriering the channel with OFDM, different subcarrier resources are allocated to different users to achieve multiple access. Consider a downlink OFDMA network jointly processed by multiple base stations, which contains $k$ cooperative cells, and $M$ users are evenly distributed in each cell, as shown in Figure 4. In each cell, the system bandwidth is divided into a series of continuous subcarrier sets orthogonal to each other, and each subcarrier set forms a subchannel. Because the subcarriers of OFDM overlap in the frequency domain, there is no guard frequency. Users access different subchannels in different time slots to achieve multiple access. Therefore, in the ideal synchronization case, there

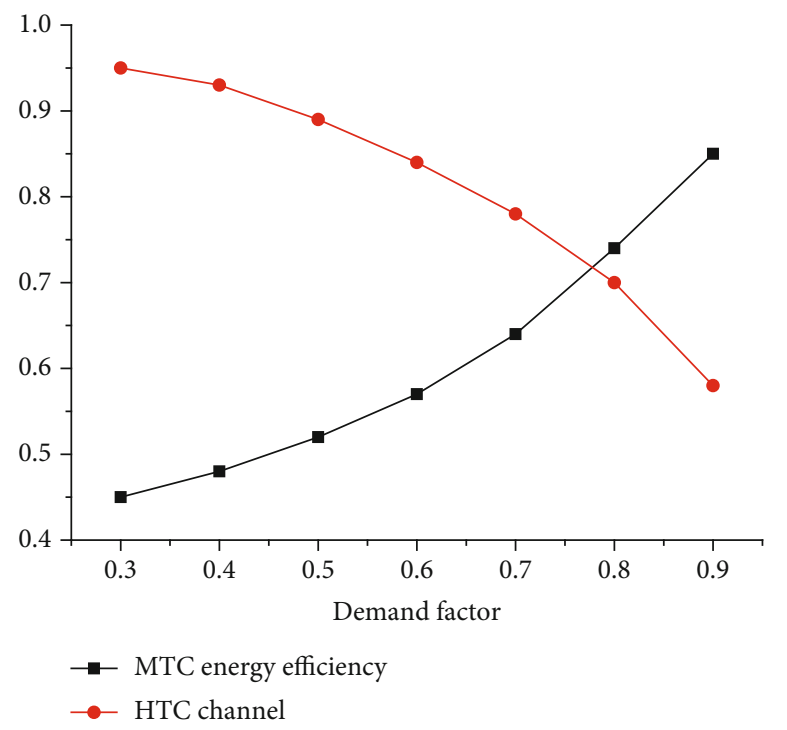

FIgURE 5: Relationship between multiobjective function and demand factor.

will be no multiuser interference in each cell. In this paper, a subchannel and a corresponding time slot are combined and defined as a basic resource allocation unit (AU). Assuming that each AU can only be occupied by a unique user, interference free multiuser access can be realized through the division of frequency and time resources.

This paper considers two different physical frame structure models in the downlink OFDMA network, namely, ordinary physical frame and superframe. Each base station adopts different operations for radio resource management within and between cells under different frame structures. 


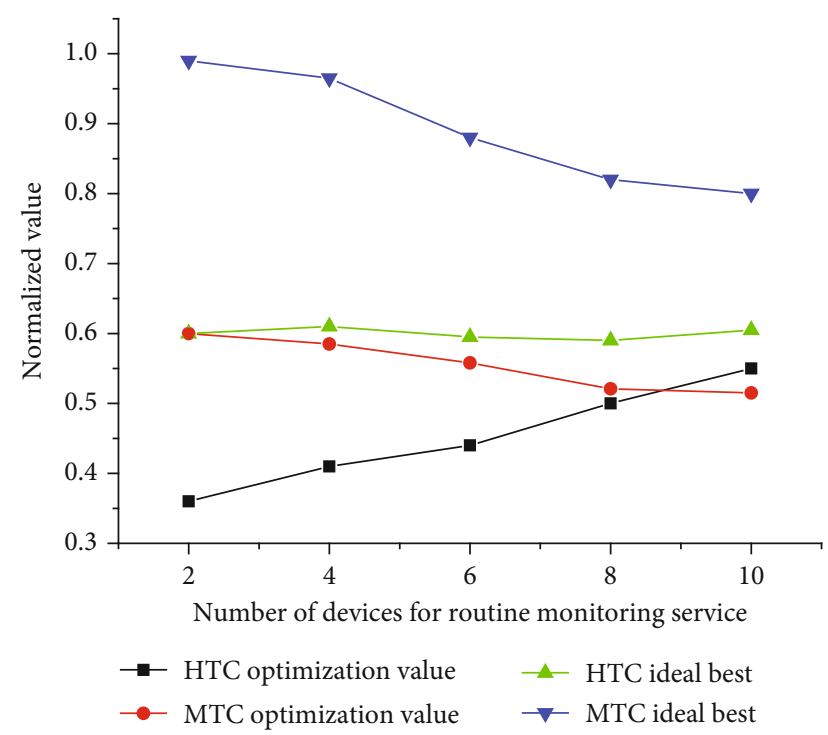

FIGURE 6: Relationship between multiobjective optimization value and the number of conventional monitoring service equipment.

Suppose that when the working frequency band is divided into several continuous subchannels, the ordinary frame is composed of these subchannels and several continuous time slots. The super physical frame is composed of several time continuous ordinary frames.

On any $\mathrm{AU}_{n}$, the signal-to-interference noise ratio received by user $m$ from its serving base station $K$ and other adjacent base stations can be expressed by the following formula.

$$
\operatorname{SINR}_{k, m}^{n}=\frac{\left|h_{k, m}^{n}\right|^{2}}{N_{0} p_{i}^{-n} \sum_{i=1}^{K} p_{i}^{n}\left|h_{i, m}^{n}\right|^{2}},
$$

where $p$ represents the power of the transmitting base station $K$ on the resource allocation unit $\left(\mathrm{AU}_{n}\right)$ and $N_{0}$ represents the power of the additive white Gaussian noise (AWGN). $H_{\text {nim }}$ represents the complex channel response coefficient of the cofrequency interference channel on the $\mathrm{AU}_{n}$ from the base station master to the user $m$ in cell $K$.

When the base station performs equal power allocation among $\mathrm{AU}$ in the physical frame, there are

$$
p_{t}=N p_{k}^{n}
$$

where $p$ represents the total transmission power of each base station and $N$ represents the total number of radio resource allocation units (AU) contained in each physical frame.

Therefore, for the scheduling user $m$ working on the $\mathrm{AU}_{n}$ in cell $K$, the downlink user rate can be expressed as follows:

$$
r_{k, m}^{n}=\log _{2}\left(1+\frac{\left|h_{k, m}^{n}\right|^{2}}{N_{0} p_{i}^{-n} \sum_{i=1}^{K} p_{i}^{n}\left|h_{i, m}^{n}\right|^{2}}\right) .
$$

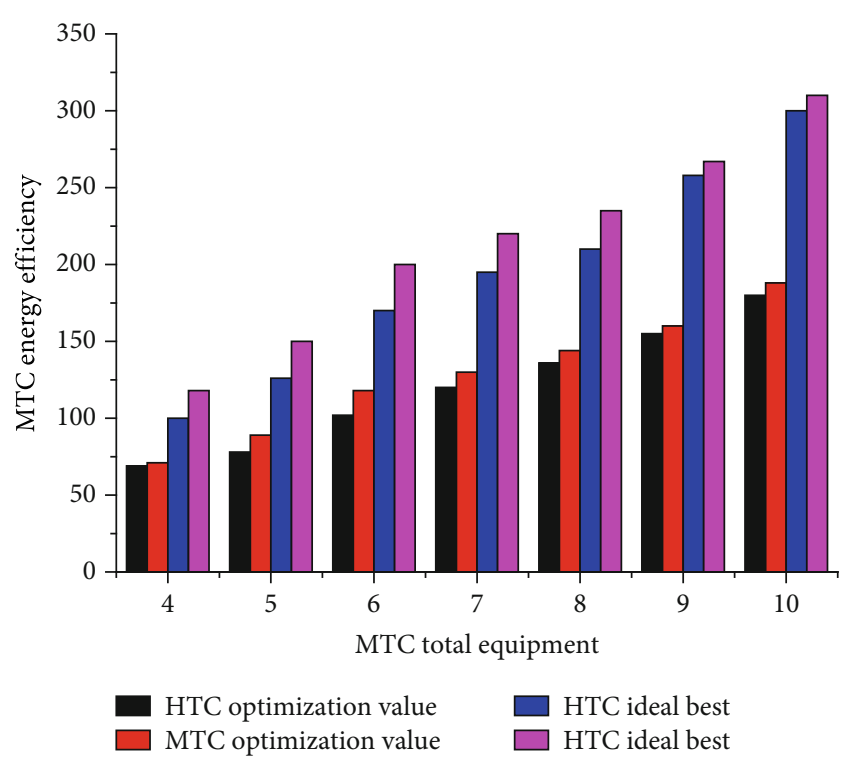

FIGURE 7: Relationship between multiobjective optimization value and total number of MTC equipment.

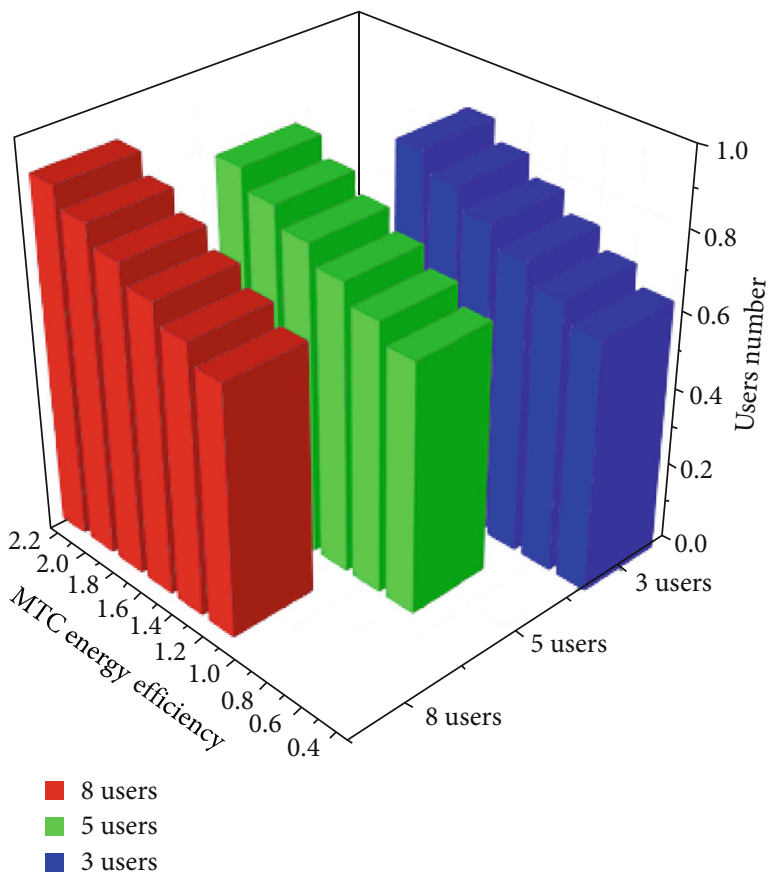

Figure 8: Pareto optimal solution.

Further, the total rate of scheduling user $m$ in cell $K$ can be expressed as

$$
R_{k, m}^{n}=\sum_{n \in N} \log _{2}\left(1+\operatorname{SINR}_{k, m}^{n}\right)
$$

where NT, $m$ represents the AU set composed of all resource allocation units occupied by user $m$ in cell $K$.

By establishing intercell cooperative links to build a multicell joint signal processing system, interference can be eliminated and cooperation gain can be obtained. Therefore, 


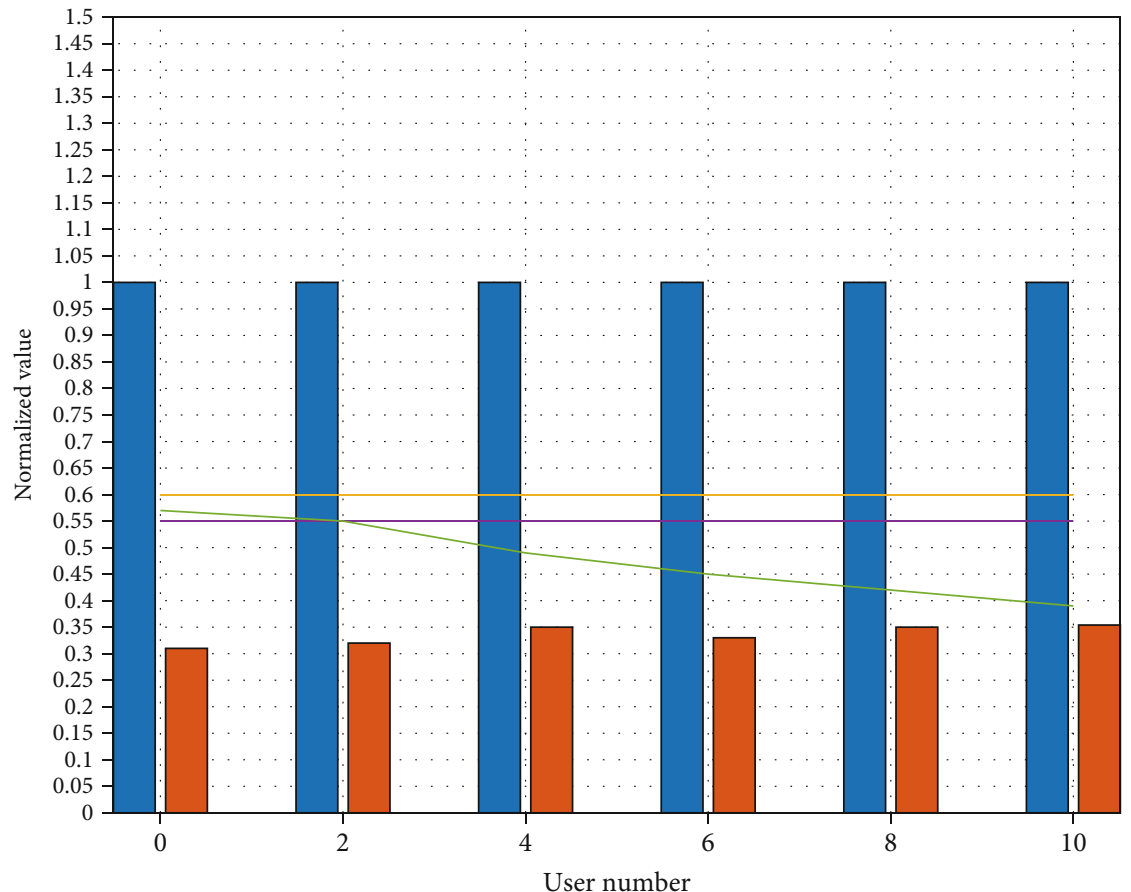

$\square$ MTC energy efficiency algorithm proposed in this paper
$\square$ MTC ENB interference algorithm proposed in this paper
HTC channel capacity weighted sum algorithm
MTC energy efficiency weighted sum algorithm
MTC ENB interference weighted sum algorithm

FIGURE 9: Relationship between normalized values of three objective functions and the number of users.

the network capacity gain of establishing cooperative links can be modeled as follows:

$$
\begin{aligned}
\text { benefit } & =\frac{\widehat{r}_{k, m}^{n}-r_{k, m}^{n}}{\widehat{r}_{k, p}^{n}}, \\
\widehat{r}_{k, m}^{n} & =\log _{2}\left(\frac{p_{k}^{n}\left|h_{k, m}^{n}\right|^{2}}{p_{i}^{n} \sum_{i=1}^{K} p_{i}^{n}\left|h_{i, m}^{n}\right|^{2}+N_{0}}\right), \\
r_{k, m}^{n} & =\log _{2}\left(\frac{p_{k}^{n}\left|h_{k, m}^{n}\right|^{2}}{\sum_{i=1}^{K} p_{i}^{n}\left|h_{i, m}^{n}\right|^{2}+N_{0}}\right) .
\end{aligned}
$$

\section{Results and Discussion}

4.1. Performance Analysis in Orthogonal Resource Scenarios. The relationship between multiobjective function and demand factor $p$ is simulated in Figure 5. In order not to lose generality, the energy efficiency of MTC and the channel capacity of HTC are normalized, respectively. The channel varies randomly, and the values vary from large to small, so the normalization of channel coefficients is for better data comparison. The number of three services of MTC equipment is $\left(n_{\mathrm{SM}}, n_{\mathrm{EA}}, n_{\mathrm{RM}}\right)=(4,4,2)$. As shown in Figure 5, when the demand factor $p$ changes from 0.3 to 0.9 , the energy efficiency of the normalized MTC equipment continues to increase. This is because the higher the weight factor of MTC devices, the higher their priority to obtain band resources. On the contrary, the channel capacity of the normalized HTC device decreases with the increase in $P$. This is because fewer subcarrier resources are allocated to HTC devices. It is worth noting that even when $p$ is very high, the normalized energy efficiency value will not increase sharply, which is conducive to protecting the QoS of HTC equipment from changing too fast.

The relationship between MTC energy efficiency and HTC energy efficiency and the number of equipment users served by RM is discussed in Figure 6. The increasing number of users means that the overall QoS requirements of the MTC system are improving. Therefore, it can be seen from the curve in the figure that as the proportion of service devices gradually increases, the bandwidth available for HTC devices gradually decreases. Therefore, the optimal value of HTC channel capacity calculated by the KM algorithm and its ideal point are in a downward trend. In addition, by observing the curve in the figure, we can know that the optimal value of MTC channel capacity and its ideal point are also in a downward trend. This is because MTC equipment improves its ability to obtain power resources to meet high QoS requirements, thus reducing the energy efficiency value of the system. As a comparison with the algorithm in this paper, the performance of the weighted sum (WS) algorithm is also reflected in this figure. Assuming that the weight ratio of the two objective functions of the weighted sum algorithm is also $1: 1$, it can be seen from the comparison curve in the figure that when the weighted 


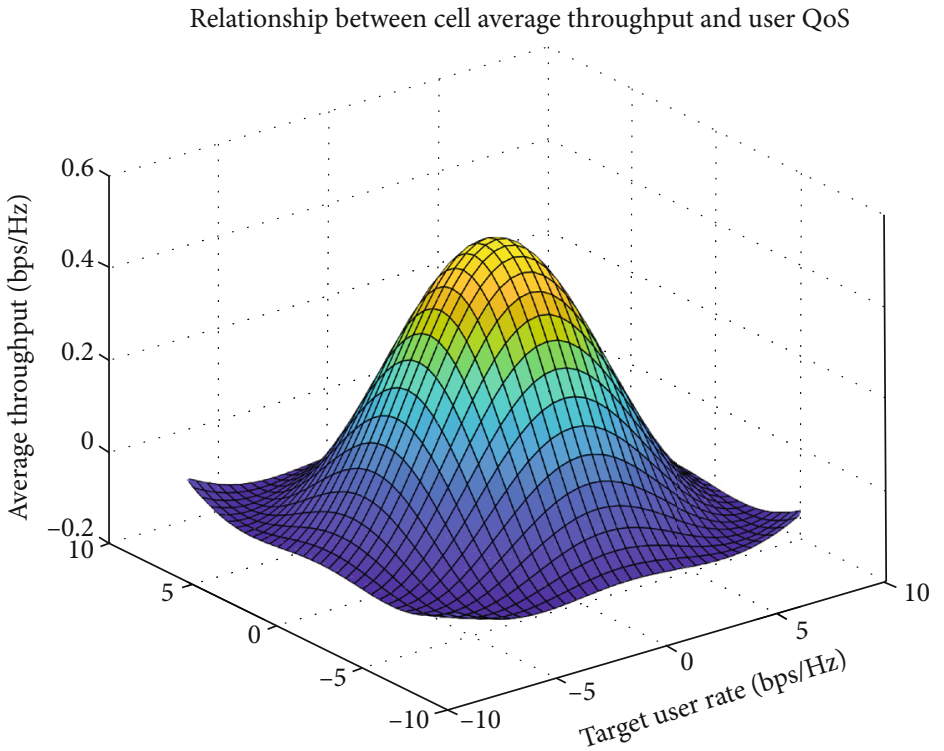

(a) Relationship between cell average throughput and user QoS Relationship between user satisfaction and user QoS

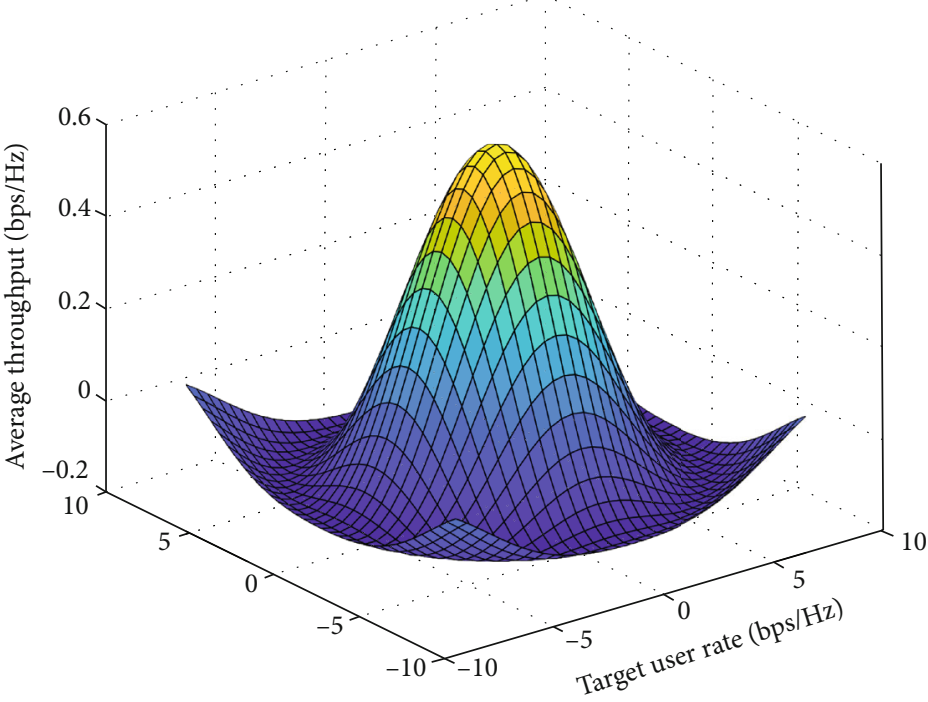

(b) Relationship between user satisfaction and user QoS

FIgURE 10: Performance analysis of multiple indicators.

sum algorithm is adopted, the MTC energy efficiency and HTC channel capacity curve decrease or increase significantly with the change of the number of devices. Using this algorithm, we can see that the fluctuation range of these two curves is not large. The weighted Chebyshev method is an iterative multivariable optimization method based on the Remez exchange algorithm, which can be used for polynomial interpolation. The corresponding interpolation polynomials can minimize the Runge phenomenon and provide the best uniform approximation of polynomials in continuous functions. It can be seen that the weighted Chebyshev algorithm adopted in this paper to solve the multiobjective optimization problem can better reflect the fairness of the compromise between the two objective functions than the weighted sum algorithm, which is more in line with the scene in the actual network.
The effectiveness of the proposed multiobjective optimization algorithm under different user equipment scenarios is proven in Figure 7. It can be seen from the figure that with the increasing number of users $n$, the curve of the ideal point and the optimization curve obtained by the KM algorithm are in an increasing trend. Therefore, in the scenario of different numbers of users, the multiobjective optimization algorithm proposed in this paper still has good applicability, which is more suitable for the actual needs of future wireless networks.

Figure 8 shows the trade-off between energy efficiency and channel capacity in the scenario of 8 users, 5 users, and 3 users. When the demand factor $P$ changes from 0.65 to 0.9 , the Pareto optimal solution of HTC equipment and MTC equipment changes. The Pareto optimal state is that there is no more room for Pareto improvement; that is, 
Relationship between average network throughput and user QoS

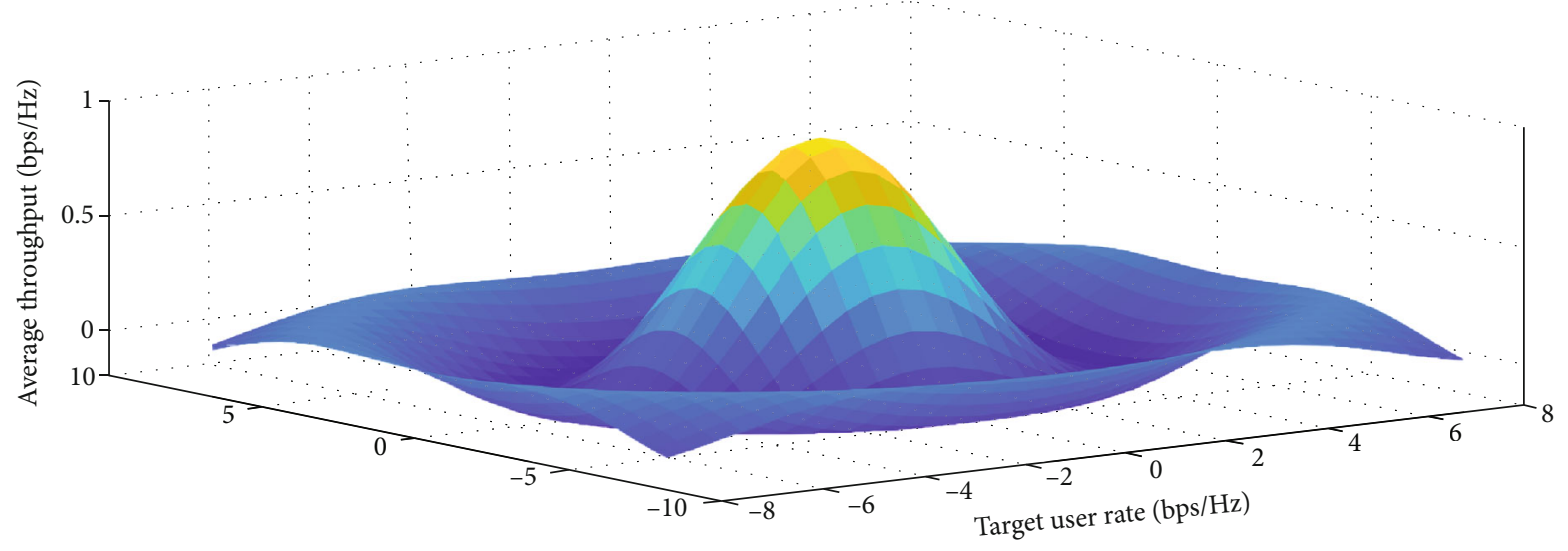

(a) Relationship between average network throughput and user QoS

Relationship between backhaul network capacity consumption and user QoS

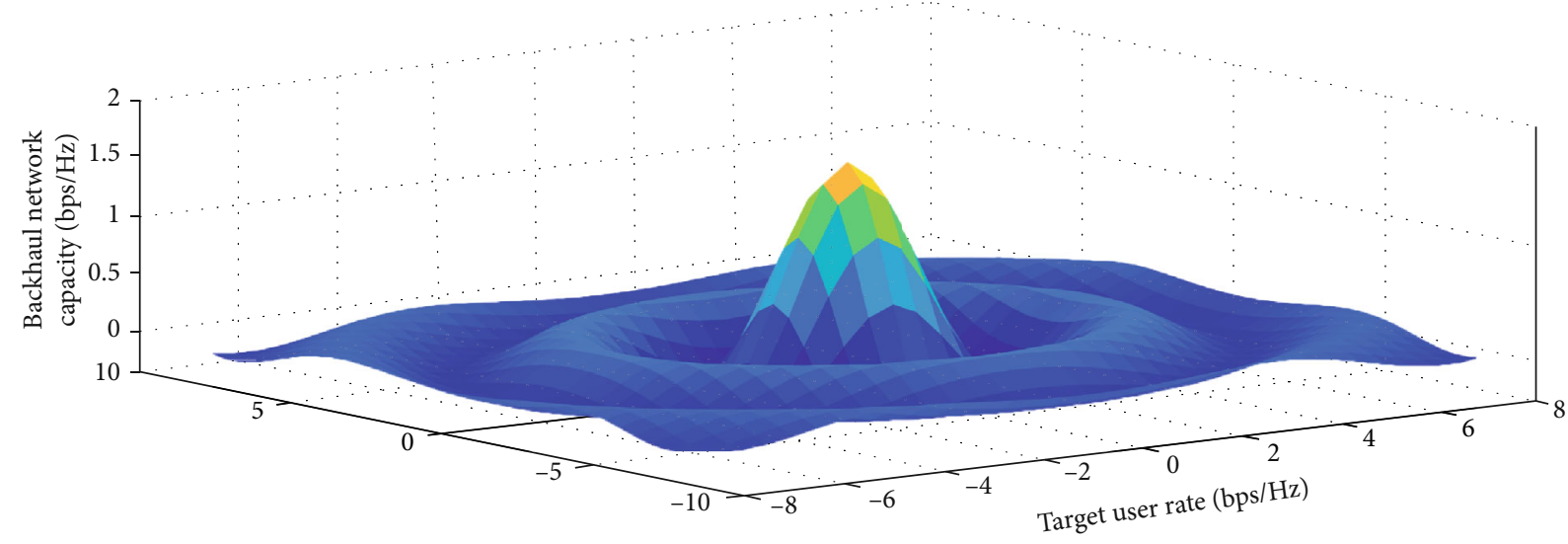

(b) Relationship between backhaul network capacity consumption and user QoS

FIGURE 11: Relationship between average network throughput performance and backhaul network capacity consumption and user requirements.

Pareto improvement is the path and method to achieve the Pareto optimal. The curve of the former shows a downward trend, and the curve of the latter shows an upward trend. This is because with the increase in $P$, MTC devices have higher priority in obtaining bandwidth and spectrum resources. Similarly, when $p$ decreases, the curve in the graph will show the opposite trend. In short, in the actual scenario, according to the demand factor of MTC equipment, network managers can balance the system performance of MTC and HTC by adjusting the weight function.

4.2. Performance Analysis under the Interference Scenario. In performance analysis under the interference scenario in Figure 9, the performance of the weighted Chebyshev algorithm used in this paper is compared with that of the traditional weighted sum algorithm, the weight factor is set as $\Phi_{1}=\Phi_{2}=\Phi_{3}=0.33$, and the algorithm of three targets is normalized. As can be seen from the figure, when the weighted Chebyshev algorithm is adopted, the normalized value of MTC interference to ENB is reduced by $2 \%$. In contrast, for different numbers of users, when using the weighted sum algorithm, the optimal channel capacity of
HTC equipment is close to its ideal point value 1 , which is much higher than that obtained by using the weighted Chebyshev algorithm. However, the optimization performance of MTC equipment is much lower than that of the weighted Chebyshev algorithm.

4.3. Optimized Performance Analysis. Figure 10(a) shows the relationship between the change of user QoS requirements and the change of network average throughput and compares the impact of the change of cell average number of users on throughput. It can be seen that with the increase in user QoS requirements, the average throughput of the cell also increases gradually. However, when the average number of users per cell increases, the average cell throughput decreases. Moreover, with the increase in user QoS requirements, this difference becomes more and more obvious. This is because there are too many users, resulting in fierce competition among users in the cell, and the available wireless resources are quickly allocated, making it difficult to maintain user demand.

Figure 10(b) shows the relationship between the change of user QoS requirements and user satisfaction in the same 
case. It can be seen that when the user QoS is generally low, most users can meet their communication quality requirements regardless of the total number of users. However, with the increase in user QoS demand, the user dissatisfaction of cells with high user density increases sharply compared with cells with low user density. Figures 10(a) and 10(b) also show that relying solely on wireless resource management and user scheduling is difficult to meet the large number of users and high user communication requirements.

Figure 11(a) describes the relationship between the average cell throughput and the user QoS requirements. The results show that with the improvement of user QoS requirements, the performance of the three algorithms shows an upward trend. The performance of the proposed algorithm is between the other two, because the proposed algorithm is limited by the backhaul network capacity, and there is a trade-off between throughput performance and backhaul network consumption. The problem of limited backhaul link capacity is common in cellular communication systems, which is also the bottleneck to realize the potential performance gain of downlink comp.

The backhaul network is the return line of the line. Generally, the end users have a large download service and a small amount of uplink data. The throughput reflects the amount of data that can actually pass through the network and receive the backhaul network capacity limit. Figure 11(b) compares the backhaul network capacity consumption of the two algorithms in Figure 11(a). The global cooperation algorithm works better under the nonconvex setting, but it depends on the global learning rate. It can be seen that with the increase in user QoS requirements, the backhaul network communication pressure caused by the global cooperation algorithm increases approximately linearly, and the consumption is too large. Relatively speaking, the algorithm proposed in this paper maintains the performance improvement of the whole network brought by cooperation.

\section{Conclusion}

Firstly, this paper considers the coexistence of M2M and $\mathrm{H} 2 \mathrm{H}$ and considers the allocation and management of band resources and power resources. A multiobjective problem is constructed for joint spectrum and power resource management, and the complex optimization problem is transformed into a single objective optimization problem that can be solved directly by the weighted Chebyshev algorithm. Secondly, in view of the additional interference caused by the introduction of $\mathrm{M} 2 \mathrm{M}$ communication, an intercell cooperative link selection algorithm is proposed, which not only eliminates the interference and improves the rate but also effectively reduces the network capacity requirements of wireless resource management. From the performance simulation results, it can be seen that under the resource management of the proposed algorithm, MTC and HTC systems can coexist fairly. Moreover, the proposed scheme shows performance advantages in user fairness, average network throughput, and communication pressure relief for the backhaul network and maximizes performance gain by minimizing network redundancy overhead.

\section{Data Availability}

The data used to support the findings of this study are available from the corresponding author upon request.

\section{Conflicts of Interest}

The authors declare that they have no known competing financial interests or personal relationships that could have appeared to influence the work reported in this paper.

\section{References}

[1] H. Dujuan, "Mobile communication technology of sports events in 5G era," Microprocessors and Microsystems, vol. 80, article 103331, 2021.

[2] S. Buffa, M. Cozzini, M. D’Antoni, M. Baratieri, and R. Fedrizzi, "5th generation district heating and cooling systems: a review of existing cases in Europe," Renewable and Sustainable Energy Reviews, vol. 104, pp. 504-522, 2019.

[3] A. Esfahani, G. Mantas, R. Matischek et al., "A lightweight authentication mechanism for M2M communications in industrial IoT environment," IEEE Internet of Things Journal, vol. 6, no. 1, pp. 288-296, 2019.

[4] Y. Mehmood, N. Haider, M. Imran, A. Timm-Giel, and M. Guizani, "M2M communications in 5G: state-of-the-art architecture, recent advances, and research challenges," IEEE Communications Magazine, vol. 55, no. 9, pp. 194-201, 2017.

[5] G. Tuna, D. G. Kogias, V. C. Gungor, C. Gezer, E. Taşkın, and E. Ayday, "A survey on information security threats and solutions for machine to machine (M2M) communications," Journal of Parallel and Distributed Computing, vol. 109, pp. 142-154, 2017.

[6] X. Liu and N. Ansari, "Resource allocation in UAV-assisted $\mathrm{M} 2 \mathrm{M}$ communications for disaster rescue," IEEE Wireless Communications Letters, vol. 8, no. 2, pp. 580-583, 2019.

[7] E. Lara, L. Aguilar, M. A. Sanchez, and J. A. García, "Lightweight authentication protocol for M2M communications of resource-constrained devices in industrial internet of things," Sensors, vol. 20, no. 2, p. 501, 2020.

[8] M. Kamel, W. Hamouda, and A. Youssef, "Uplink performance of NOMA-based combined HTC and MTC in ultradense networks," IEEE Internet of Things Journal, vol. 7, no. 8, pp. 7319-7333, 2020.

[9] V. Mancuso, P. Castagno, M. Sereno, and M. A. Marsan, "Modeling MTC and HTC radio access in a sliced 5G base station," IEEE Transactions on Network and Service Management, vol. 18 , no. 2, pp. 2208-2225, 2021.

[10] D. Tokody and F. Flammini, "The intelligent railway system theory," International Transportation, vol. 69, no. 1, pp. 3840, 2017.

[11] R. P. Centelles, F. Freitag, R. Meseguer, and L. Navarro, "Beyond the star of stars: an introduction to multihop and mesh for LoRa and LoRaWAN," IEEE Pervasive Computing, vol. 20, no. 2, pp. 63-72, 2021.

[12] A. Aijaz, M. Tshangini, M. R. Nakhai, X. Chu, and A.-H. Aghvami, "Energy-efficient uplink resource allocation in LTE networks with $\mathrm{M} 2 \mathrm{M} / \mathrm{H} 2 \mathrm{H}$ co-existence under statistical QoS guarantees," IEEE Transactions on Communications, vol. 62, no. 7, pp. 2353-2365, 2014. 
[13] H. Yang and S. An, "Robustness evaluation for multi-subnet composited complex network of urban public transport," Alexandria Engineering Journal, vol. 60, no. 2, pp. 2065-2074, 2021.

[14] M. A. Mehaseb, Y. Gadallah, A. Elhamy, and H. Elhennawy, "Classification of LTE uplink scheduling techniques: an M2M perspective," IEEE Communications surveys \& tutorials, vol. 18, no. 2, pp. 1310-1335, 2016.

[15] K. Zheng, F. Hu, W. Wang, W. Xiang, and M. Dohler, "Radio resource allocation in LTE-advanced cellular networks with M2M communications," IEEE Communications Magazine, vol. 50, no. 7, pp. 184-192, 2012.

[16] S. Hamdoun, A. Rachedi, and Y. Ghamri-Doudane, "Graphbased radio resource sharing schemes for MTC in D2Dbased 5G networks," Mobile Networks \& Applications, vol. 25, no. 3, pp. 1095-1113, 2020.

[17] H. S. Jang, H. S. Park, and D. K. Sung, "A non-orthogonal resource allocation scheme in spatial group based random access for cellular M2M communications," IEEE Transactions on Vehicular Technology, vol. 66, no. 5, pp. 4496-4500, 2016.

[18] C. Yin, L. Shi, R. Sun, and J. Wang, "Improved collaborative filtering recommendation algorithm based on differential privacy protection," The Journal of Supercomputing, vol. 76, no. 7, pp. 5161-5174, 2020.

[19] D. Kim, D. Hyeon, J. Oh, W.-S. Han, and H. Yu, "Influence maximization based on reachability sketches in dynamic graphs," Information Sciences, vol. 394-395, pp. 217-231, 2017.

[20] Y.-H. Lin, J.-J. Huang, C.-I. Fan, and W.-T. Chen, "Local authentication and access control scheme in M2M communications with computation offloading," IEEE Internet of Things Journal, vol. 5, no. 4, pp. 3209-3219, 2018.

[21] G. R. MacCartney and T. S. Rappaport, "Millimeter-wave base station diversity for $5 \mathrm{G}$ coordinated multipoint (CoMP) applications," IEEE Transactions on Wireless Communications, vol. 18, no. 7, pp. 3395-3410, 2019.

[22] Y. J. Chun, S. L. Cotton, H. S. Dhillon, F. J. Lopez-Martinez, J. F. Paris, and S. K. Yoo, "A comprehensive analysis of 5G heterogeneous cellular systems operating over $\kappa-\mu$ shadowed fading channels," IEEE Transactions on Wireless Communications, vol. 16, no. 11, pp. 6995-7010, 2017.

[23] F. Wu, R. Tan, C. Zhang et al., "Mixed numerology interference recognition approach for 5G NR," IEEE Wireless Communications Letters, vol. 10, no. 10, pp. 2135-2139, 2021.

[24] K. S. Ali, H. Elsawy, A. Chaaban, and M.-S. Alouini, "Nonorthogonal multiple access for large-scale 5G networks: interference aware design," IEEE Access, vol. 5, pp. 21204-21216, 2017.

[25] F. Qamar, M. H. D. N. Hindia, K. Dimyati, K. A. Noordin, and I. S. Amiri, "Interference management issues for the future $5 \mathrm{G}$ network: a review," Telecommunication Systems, vol. 71, no. 4, pp. 627-643, 2019.

[26] M. Abdullah, S. H. Kiani, and A. Iqbal, "Eight element multiple-input multiple-output (MIMO) antenna for 5G mobile applications," IEEE Access, vol. 7, pp. 134488-134495, 2019.

[27] M. S. Sharawi, "Current misuses and future prospects for printed multiple-input, multiple-output antenna systems wireless corner," IEEE Antennas and Propagation Magazine, vol. 59, no. 2, pp. 162-170, 2017.
[28] E. Chen, M. Tao, and Y. F. Liu, "Joint base station clustering and beamforming for non-orthogonal multicast and unicast transmission with backhaul constraints," IEEE Transactions on Wireless Communications, vol. 17, no. 9, pp. 6265-6279, 2018.

[29] J. An, Y. Zhang, X. Gao, and K. Yang, "Energy-efficient base station association and beamforming for multi-cell multiuser systems," IEEE Transactions on Wireless Communications, vol. 19, no. 4, pp. 2841-2854, 2020.

[30] F. Liu, C. Masouros, A. Li, H. Sun, and L. Hanzo, "MU-MIMO communications with MIMO radar: from co-existence to joint transmission," IEEE Transactions on Wireless Communications, vol. 17, no. 4, pp. 2755-2770, 2018. 